\title{
A importância do pré-natal no diagnóstico e tratamento da sífilis congênita
}

\author{
The importance of prenatal care in the diagnosis and treatment of congenital syphilis
}

\author{
La importancia de la atención prenatal en el diagnóstico de la sífilis congénita
}

Vitoria Vilas Boas da Silva Bomfim ${ }^{1 *}$, Maria Eduarda Lopes de Macedo Bezerra², Brenda Tayrine Tavares Souza ${ }^{3}$, Folve Ariel Garcia Alencar ${ }^{4}$, Yesly Marinho da Rocha Barreto ${ }^{5}$, Angelica Ribeiro do Nascimento Oliveira ${ }^{6}$, Monalisa Batatinha de Castro Silva ${ }^{7}$, Emily da Silva Eberhardt ${ }^{8}$, Gleice Matos Guimarães ${ }^{9}$, Elen Gomes de Oliveira ${ }^{9}$.

\section{RESUMO}

Objetivo: Examinar as formas de transmissão vertical da sífilis com o intuito de adotar medidas preventivas, diagnósticas e terapêuticas em pacientes gestantes. Métodos: Revisão integrativa de literatura realizada nas bases de dados SciELO, LILACS, BDENF e MEDLINE, através dos seguintes DECS: "sífilis congênita" e "diagnóstico pré-natal". Incluídos artigos disponíveis, nos idiomas português, espanhol e inglês, que abordassem a temática, nos últimos dez anos. Resultados: Houve um aumento nos casos de sífilis gestacional e sífilis congênita (SC), uma taxa média de detecção de 20,1 casos/1000 nascidos vivos para SG e de 5,7 casos/1000 nascidos vivos para SC, com predomínio para população vulnerável. Há fatores que influenciam no diagnóstico tardio e na baixa adesão ao tratamento, por isso faz-se necessário atuar em diferentes esferas para combater a sífilis e sendo a melhor medida de prevenção a SC a realização do pré-natal adequado onde as gestantes realizaram seis ou mais consultas. Considerações finais: As medidas de prevenção a serem tomadas, são diagnóstico rápido, educação em saúde, o uso de método contraceptivo de barreira, tratamento completo durante a descoberta, tornando assim mais difícil a transmissão por via transplacentária.

Palavras-chave: Sífilis, Sífilis congênita, Pré-natal, Transmissão vertical.

\section{ABSTRACT}

Objective: To examine the forms of vertical transmission of syphilis in order to adopt preventive, diagnostic and therapeutic measures in pregnant patients. Methods: Integrative literature review carried out in the SciELO, LILACS, BDENF and MEDLINE databases, through the following DECS: "congenital syphilis" and "prenatal diagnosis". Available articles in Portuguese, Spanish and English that addressed the theme, in the last ten years. Results: There was an increase in cases of gestational syphilis and congenital syphilis (CS), with an average detection rate of 20.1 cases/1000 live births for SG and 5.7 cases/1000 births There are factors that influence late diagnosis and poor adherence to treatment, so it is necessary to act in different spheres to fight syphilis, and the best measure of prevention for CS is to carry out the adequate prenatal care where the pregnant women had six or more consultations. Final considerations: The preventive measures to be taken are rapid diagnosis, health education, the use of barrier contraceptive method, complete treatment during discovery, thus making transplacental transmission more difficult.

Keywords: Syphilis, Congenital syphilis, Prenatal, Vertical transmission.

\section{RESUMEN}

Objetivo: Examinar las formas de transmisión vertical de la sífilis para adoptar medidas preventivas, diagnósticas y terapéuticas en pacientes embarazadas. Métodos: Revisión integrativa de la literatura realizada en las bases de datos SciELO, LILACS, BDENF y MEDLINE, a través de las siguientes DECS: "sífilis congénita" y "diagnóstico prenatal". Artículos disponibles en portugués, español e inglés que abordaron el tema, en los últimos diez. Resultados: Hubo un aumento de casos de sífilis gestacional y sífilis congénita (SC), con una tasa de detección promedio de 20,1 casos / 1000 nacidos vivos para SG y 5,7 casos / 1000 nacimientos Hay factores que influyen en el diagnóstico tardío y la mala adherencia al tratamiento, por lo que es necesario actuar en diferentes ámbitos para combatir la sífilis, y la mejor medida de prevención del SC es realizar la adecuada atención prenatal donde la gestante tuvo seis o más consultas. Consideraciones finales: Las medidas preventivas a tomar son el diagnóstico rápido, la educación sanitaria, el uso de método anticonceptivo de barrera, el tratamiento completo durante el descubrimiento, dificultando así la transmisión transplacentaria.

Palabras clave: Sífilis, Sífilis congénita, Prenatal, Transmisión vertical.

\footnotetext{
${ }^{1}$ Centro Universitário Jorge Amado (UNIJORGE), Salvador - BA. *E-mail: pesquisaclinica9@gmail.com

${ }^{2}$ Faculdade Mineira (UNIMINAS), Juiz de Fora - MG.

${ }^{3}$ Centro Universitário do Distrito Federal (UDF), Brasília - DF.

${ }^{4}$ Universidade do Estado do Pará (UEPA), Belém - PA.

${ }^{5}$ Centro Universitário Brasileiro (UNIBRA), Recife - PE.

${ }^{6}$ Centro Universitário Maurício de Nassau (UNINASSAU), Teresina - PI.

${ }^{7}$ Universidade do Estado da Bahia (UNEB), Senhor do Bomfim - BA.

${ }^{8}$ Centro Universitário Cenecista de Osório (UNICNEC), Osório - RS.

${ }^{9}$ Faculdade Pitágoras de Teixeira de Freitas (PIT-TEIXEIRA), Teixeira de Freitas - BA.
}

SUBMETIDO EM: 6/2021

PUBLICADO EM: 7/2021 


\section{INTRODUÇÃO}

Sífilis congênita é caracterizada pela disseminação hematogênica do Treponema Pallidum da gestante não tratada ou inadequadamente tratada para o feto através da via transplacentária. A transmissão do treponema da mãe para o feto pode ocorrer em qualquer etapa gestacional tendo como principais fatores de transmissibilidade vertical o estado sifilítico da gestante e o tempo de exposição do feto. Quanto mais recente a contaminação, maior o número de treponemas circulantes no organismo e maior a chance de transmissão para o feto (BRASIL, 2015; MAGALHÃES DMS, et al., 2013).

Existem outras formas de contaminação da sífilis congênita, sendo elas de forma direta. A primeira delas ocorre no momento em que a criança atravessa pelo canal de parto e a genitora sifilítica possui nesta região lesões genitais. A segunda maneira se dá por meio do aleitamento materno, sendo nesse caso que a mãe já apresenta uma lesão mamária causada pela sífilis. As manifestações da sífilis na criança são complexas já que muitas das vezes são assintomáticos, são classificados em sífilis precoce e tardia. A precoce aparece até os dois anos de vida, a tardia se apresenta após o segundo ano de vida. Em 2002, o Programa de Humanização no pré-natal e Nascimento (PHPN) foi implementado pelo Ministério da Saúde com objetivo de facilitar o acesso e a qualidade do atendimento ao pré-natal, parto e puerpério, reduzindo as taxas de morbimortalidade materna e do recém-nascido no Brasil (BRASIL, 2015; CAVALCANTE PAM, et al., 2017).

No momento que a gestante decide procurar o atendimento médico e se submeter ao acompanhamento do pré-natal ela acaba de certa forma permitindo que outras pessoas cuidem do seu corpo, que é algo tão íntimo, delicado e pessoal, e consequentemente da nova vida que está sendo gerada em seu útero. Respeitar essa fase única da vida de toda mulher demandado profissional de saúde, além do saber técnico, habilidades que o capacitem para melhor acolher essa paciente e prestar um serviço de qualidade. Ouvir atentamente o que a paciente tem a dizer, dirimir dúvidas, orientar sobre procedimento e comportamentos, dentre outros questionamentos, configura como dever do profissional de saúde perante a paciente (LAFETÁ KRG, et al., 2016).

Quando diagnosticada em tempo hábil, a sífilis pode ser tratada e seus efeitos no feto podem ser eliminados ou minimizados. A sífilis congênita é considerada uma doença de fácil prevenção desde que a gestante infectada seja devidamente tratada. Atualmente provas sorológicas como IGG, IGM e Veneral Diseases Research Laboratory (VDRL) são realizadas em pacientes suspeitos de sífilis com intuito de poder identificar cada vez mais precocemente a doença e conseguir adotar as medidas profiláticas adequadas (SUTO CSS, et al., 2016).

Durante o pré-natal, a realização da pesquisa do VDRL é de caráter obrigatório. Ademais, toda gestante que for admitida no hospital ou realizar o parto deverá também submeter-se ao teste VRDL. Na criança recém-nascida alguns critérios objetivos devem ser respeitados no momento da execução do VDRL. Em recém-nascidos, cuja genitora seja considerada positiva para sífilis na ocasião do parto ou com história prévia da doença, ou quando a gestante é portadora de sífilis não tratada ou tratada inadequadamente durante o pré-natal, independente do resultado do VDRL, a criança é obrigada a realizar o teste do VDRL. O tratamento padrão ouro para sífilis é penicilina aplicada em 2.400.000 UI, intramuscular, uma vez por semana durante três semanas. Já em neonatos o tratamento é penicilina $50.000 \mathrm{Ul} / \mathrm{Kg} / \mathrm{dose}$, endovenosa, a cada 12 horas nos primeiros sete dias de vida e a cada 8 horas após os sete dias de vida por dez dias (LAFETÁ KRG, et al., 2016; FIGUEIREDO DCMM, et al., 2020).

A prestação de assistência de qualidade durante toda a gestação e puerpério é a melhor forma de combater os casos de transmissão vertical da sífilis. A sífilis é uma doença de notificação compulsória no Brasil. O aumento do número de casos se deu pelo baixo nível socioeconômico que está associado à baixa escolaridade, causando assim uma falta de entendimento sobre as IST's e os cuidados a serem tomados no pré-natal. Em específico nós casos de sífilis podemos incluir a falta de realização de exames diagnósticos, falta de conhecimento dos profissionais para reconhecer os sinais e sintomas da doença ainda no início, bem como o erro na interpretação de sorologias e testes rápidos, dificuldade no tratamento da mãe e do parceiro, bem como o tratamento incompleto, além de um pré-natal inadequado e incompleto (GUANABARA MAO, et al., 2017). 
Sabe-se, que a assistência no pré-natal estendida a todas as grávidas seria a maneira mais confiável e segura de se eliminar a sífilis materna e suas consequências e complicações durante a gestação. Entendese ainda a necessidade real de informação e esclarecimentos às grávidas e seus familiares mais próximos sobre a gravidade e consequências que podem ser causadas pela mesma (BRASIL, 2015).

Por determinação do Ministério da saúde todas a gestantes durante a primeira consulta de pré-natal ou durante o primeiro trimestre da gestação deve realizar o teste para Sífilis (teste rápido para a triagem da sífilis e/ou VDRL) que é feito através de um simples exame de sangue, onde é recolhido uma amostra de sangue em pequena quantidade e analisada em laboratório, repetido no terceiro trimestre e no parto. Diante disso, afirma-se que grande parte das mulheres portadoras de Sífilis é identificada no momento da gravidez e/ou do parto (ARAUJO EC, et al., 2018).

O diagnóstico da sífilis no geral, varia em relação a fase evolutiva dessa doença, devem ser utilizados os testes não treponêmico e também testes treponêmicos. A penicilina é o padrão ouro para tratamento da sífilis, seja ela adquirida ou congênita. Ela atua impedindo que as enzimas catalisadoras atuem. Ela é um antibiótico eficaz desde que usada nas doses preconizadas e nos intervalos de tempo adequados. Se houver alergia materna à penicilina, o uso de drogas alternativas, como a eritromicina, não tratará a infecção fetal, existindo poucos estudos a respeito de drogas alternativas, como o ceftriaxone e a azitromicina, em gestantes luéticas (ARRUDA LR e RAMOS ARS, 2020).

Frente ao exposto o estudo objetiva examinar através de uma revisão integrativa as formas de transmissão vertical da sífilis com o intuito de informar sobre as medidas que podem ser adotadas para ações preventivas, diagnósticas e terapêuticas em pacientes gestantes.

\section{MÉTODOS}

Trata-se de uma revisão integrativa da literatura que consiste em seis passos para ser executada sendo eles elaboração da pergunta norteadora "Quais são as formas de transmissão vertical da sífilis e como podemos realizar prevenção, diagnóstico e tratamento em gestantes?".

A segunda etapa realizada foi buscar na literatura científica através das bases de dados Scientific Electronic Library Online (SciELO), Literatura Latino-Americana e do Caribe em Ciências da Saúde (LILACS), Banco de Dados em Enfermagem (BDENF), Medical Literature Analysis and Retrieval System Online (MEDLINE) e Revista Eletrônica Acervo Saúde através dos seguintes Descritores em Ciências da Saúde (DeCS): "sífilis congênita" e "diagnóstico pré-natal". Combinados entre si pelo operador booleano "AND" (que promove a inclusão de todos os artigos que apresentem o descritor em comum) (SOUZA MT, et al., 2010).

Como critérios de inclusão: artigos disponíveis na íntegra, nos idiomas português, espanhol e inglês, que abordassem a temática, nos últimos dez anos. Como critérios de exclusão: artigos que não contemplavam o tema e estudos repetidos nas bases de dados.

A terceira etapa realizada foi coleta de dados dos artigos científicos após aplicação dos critérios de elegibilidade, a quarta etapa foi a análise crítica e reflexiva dos estudos selecionados, foi realizado como quinta etapa a discussão dos resultados para chegarmos na sexta etapa que é a apresentação da revisão integrativa.

\section{RESULTADOS}

Foram encontrados 389 artigos, nos quais após submetidos a uma criteriosa leitura analítica, crítica e eletiva, sendo aplicados os critérios de inclusão e exclusão, restaram apenas 153 artigos a serem lidos título e resumo a fim de atender a pergunta norteadora, restando 30 artigos na qual foram lidos na íntegra sendo selecionados 16 estudos para compor a amostra final (Quadro 1). Os seus estudos foram publicados entre os anos de 2017 e 2021, sendo a maioria publicada em 2020. Todos os estudos estão em consonância com o objetivo a ser abordado no estudo. 
Quadro 1 - Síntese dos principais achados sobre Sífilis Congênita (SC).

\begin{tabular}{|c|c|c|c|}
\hline $\mathbf{N}$ & Autores (Ano) & Periódico & Principais achados \\
\hline 1 & $\begin{array}{l}\text { OZELAME JEEP, et al., } \\
2020 .\end{array}$ & $\begin{array}{c}\text { Revista de Enfermagem da } \\
\text { UFRJ. }\end{array}$ & $\begin{array}{l}\text { Estudo transversal, de caráter analítico e abordagem quantitativa. Foi coletado dados do Sistema de Informações e } \\
\text { Agravos de Notificação. Conclui-se que as condições potencializam a vulnerabilidade à sífilis congênita e gestacional, } \\
\text { sendo importante a elaboração de intervenções que minimizem as consequências de curto, médio e longo prazo. }\end{array}$ \\
\hline 2 & ALVES PIC, et al., 2020. & Ciência e Saúde Coletiva. & $\begin{array}{l}\text { Estudo quantitativo, de análise de série temporal, coletados do Sistema de Informações e Agravos de Notificação. } \\
\text { Utilizada estatística descritiva, cálculo da taxa de incidência de SC e modelo de regressão polinomial para análise da } \\
\text { tendência temporal. Constata-se no coeficiente de incidência da SC que o número de casos é crescente, necessitando } \\
\text { de uma assistência qualificada no pré-natal, diagnóstico, tratamento e ações de saúde. }\end{array}$ \\
\hline 3 & SILVA NCP, et al., 2021. & Femina. & $\begin{array}{l}\text { Estudo clínico-epidemiológico e transversal, realizado no Hospital Regional Materno Infantil de Imperatriz- MA. } \\
\text { Elaborado coleta de dados por meio de busca ativa em visitas semanais, utilizado questionário próprio e aprovado pelo } \\
\text { Comitê de Ética em Pesquisa da Universidade Federal do Maranhão. Entende-se a necessidade de atuar em diversas } \\
\text { esferas para combater e tratar a sífilis congênita, realizando ações tanto na educação e serviços de saúde, como na } \\
\text { capacitação dos profissionais atuantes. }\end{array}$ \\
\hline 4 & MACÊDO VC, et al., 2020. & Caderno de Saúde Coletiva. & $\begin{array}{l}\text { Estudo descritivo, transversal, por meio do banco de dados de um estudo caso-controle para sífilis gestacional em } \\
\text { maternidades públicas no Nordeste do Brasil, entre } 2013 \text { e } 2014 \text {. A avaliação permitiu identificar situações que } \\
\text { expressam as desiguais oportunidades de atenção à saúde de grupos vulneráveis e distintas barreiras assistenciais no } \\
\text { pré-natal que retardaram o diagnóstico e, consequentemente, o tratamento apropriado para a sífilis. Concluindo que o } \\
\text { pré-natal precoce amplia o acesso ao diagnóstico, favorecendo a identificação de mulheres expostas, implementando } \\
\text { estratégias sustentáveis de forma a contribuir mais efetivamente no controle da sífilis no país. }\end{array}$ \\
\hline 5 & $\begin{array}{l}\text { GUANABARA MAO, et al., } \\
2017 \text {. }\end{array}$ & Revista de Salud Pública. & $\begin{array}{l}\text { Pesquisa de campo realizada em quatro unidades primárias de saúde com casos múltiplos que analisou a atenção pré- } \\
\text { natal de gestantes com sífilis, identificando os aspectos que dificultaram o acesso das mesmas às tecnologias leves, } \\
\text { leves-dura e dura em saúde que podem contribuir para a prevenção da SC. Conclui-se que o acesso a diferentes } \\
\text { tecnologias para prevenção e controle da SC não é viabilizada para gestantes com sífilis. }\end{array}$ \\
\hline 6 & $\begin{array}{l}\text { DOMINGUES CSB, et al., } \\
2021 .\end{array}$ & $\begin{array}{l}\text { Epidemiologia e Serviços de } \\
\text { Saúde. }\end{array}$ & $\begin{array}{l}\text { Estudo clínico-epidemiológico elaborado com base em evidências científicas e validado em discussões com } \\
\text { especialistas. Analisa aspectos clínicos e epidemiológicos bem como recomendações aos gestores no manejo } \\
\text { programático e operacional da sífilis. Incluindo orientações para os profissionais de saúde na triagem, diagnóstico e } \\
\text { tratamento. }\end{array}$ \\
\hline 7 & $\begin{array}{l}\text { FIGUEIREDO DCMM, et al., } \\
2020 .\end{array}$ & Cadernos de Saúde Pública. & $\begin{array}{l}\text { Estudo de rastreamento, descritivo e ecológico analisando as incidências desses agravos. A amostra do estudo foi } \\
\text { composta por municípios com população acima de } 20.000 \text { habitantes, com cobertura da atenção básica superior a } 50 \% \\
\text { e nos quais a maioria das equipes foi avaliada no segundo ciclo do Programa de Melhoria do Acesso e da Qualidade } \\
\text { na Atenção Básica. Diante da análise, os dados sugerem associação entre a ampliação do acesso e redução da } \\
\text { transmissão vertical no grupo de municípios avaliados, desenvolvendo tecnologias leves e leveduras que possibilitem } \\
\text { melhor adesão ao tratamento. }\end{array}$ \\
\hline
\end{tabular}




\begin{tabular}{|c|c|c|c|}
\hline $\mathbf{N}$ & Autores (Ano) & Periódico & Principais achados \\
\hline 8 & PEREIRA BB, et al., 2020. & $\begin{array}{l}\text { Revista de Enfermagem da } \\
\text { UFSM. }\end{array}$ & $\begin{array}{l}\text { Pesquisa qualitativa realizada em um município do sul do Brasil. Os dados foram coletados em } 2018 \text { por entrevistas } \\
\text { semiestruturadas e submetidos à Análise de Conteúdo. Observa-se a importância da equipe de enfermagem na } \\
\text { realização do pré-natal e do teste rápido da sífilis. }\end{array}$ \\
\hline 9 & GOMES NS, et al., 2021. & $\begin{array}{l}\text { Revista Brasileira de Promoção } \\
\text { a Saúde. }\end{array}$ & $\begin{array}{l}\text { Pesquisa descritiva com abordagem qualitativa, desenvolvida por intermédio de entrevista semiestruturada com } \\
\text { gestantes em uma Unidade de Atenção Primária à Saúde no Rio Grande do Sul, no ano de } 2019 \text {. Percebeu-se, que as } \\
\text { gestantes demonstram pouco conhecimento em relação a sífilis gestacional e as repercussões da síflis congênita, } \\
\text { evidenciando a ausência de informação recebida durante o pré-natal. }\end{array}$ \\
\hline 10 & FARIAS RO, et al., 2020. & $\begin{array}{l}\text { Jornal Brasileiro de Doenças } \\
\text { Sexualmente Transmissíveis. }\end{array}$ & $\begin{array}{l}\text { Estudo transversal, retrospectivo e descritivo, realizado por meio de coleta de dados dos casos de sífilis gestacional e } \\
\text { congênita dos anos de } 2007-2019 \text {, no Sistema de Informação de Agravos de Notificações (SINAN). Evidenciou-se, um } \\
\text { aumento significativo do número de casos notificados de sífilis gestacional nos últimos } 13 \text { anos, além de uma alta } \\
\text { porcentagem de óbitos infantis em decorrência da doença. }\end{array}$ \\
\hline 11 & $\begin{array}{l}\text { SOUZA MHT e BECK EQ, } \\
2019 .\end{array}$ & $\begin{array}{l}\text { Revista de Enfermagem da } \\
\text { UFSM. }\end{array}$ & $\begin{array}{l}\text { Pesquisa qualitativa e descritiva realizada por meio de entrevista individual com mães de crianças portadoras de sífilis } \\
\text { congênita, desenvolvida em um hospital do Distrito Federal no ano de } 2017 \text {. Os dados mostram falta de } \\
\text { aconselhamento às gestantes a respeito da doença durante a realização do pré-natal, indicando falhas no diagnóstico e } \\
\text { intervenção do agravo. }\end{array}$ \\
\hline 12 & ARAUJO SR, et al., 2020. & $\begin{array}{l}\text { Revista Eletrônica Acervo } \\
\text { Saúde. }\end{array}$ & $\begin{array}{l}\text { Estudo qualitativo, onde demonstrou o sofrimento de cinco mães, que sofreram após hospitalização de seus recém- } \\
\text { nascidos por diagnóstico de sífilis congênita. }\end{array}$ \\
\hline 13 & $\begin{array}{l}\text { BARBOSA DFR, et al., } \\
2020 .\end{array}$ & $\begin{array}{l}\text { Revista Eletrônica Acervo } \\
\text { Saúde. }\end{array}$ & $\begin{array}{l}\text { Estudo epidemiológico realizado no SINAN, onde demonstrou que } 119 \text { casos de sífilis congênita no estado do Maceió } \\
\text { no ano de } 2019 \text {. }\end{array}$ \\
\hline 14 & ALEIXO FC, et al., 2019. & $\begin{array}{l}\text { Revista Eletrônica Acervo } \\
\text { Saúde. }\end{array}$ & $\begin{array}{l}\text { Estudo quantitativo, utilizando dados de prontuários de recém-nascidos de um hospital público no Pará, onde } 312 \\
\text { neonatos foram diagnosticados com sífilis , dentre eles } 22 \text { também apresentavam infeç̧ão por exposição ao HIV. }\end{array}$ \\
\hline 15 & NUNES IR, et al., 2020. & $\begin{array}{l}\text { Revista Eletrônica Acervo } \\
\text { Saúde. }\end{array}$ & $\begin{array}{l}\text { Estudo epidemiológico realizado no SINAN, onde demonstrou que } 9,2 \text { a cada } 1000 \text { dos nascidos vivos apresentavam } \\
\text { casos de sífilis congênita no estado do Piauí. }\end{array}$ \\
\hline 16 & SABACK MC, et al., 2019. & $\begin{array}{l}\text { Revista Eletrônica Acervo } \\
\text { Saúde. }\end{array}$ & $\begin{array}{l}\text { Pesquisa quantitativa, realizada em uma maternidade estadual de Manaus, onde } 277 \text { recém-nascidos apresentaram } \\
\text { VDRL reagente, apresentando possível caso de sífilis congênita, devido a falta ou mal realização do tratamento e do } \\
\text { pré-natal. }\end{array}$ \\
\hline
\end{tabular}

Fonte: Bomfim VVBS, et al., 2021. 
Em um estudo foi possível concluir que um aumento progressivo da sífilis gestacional (SG) e sífilis congênita (SC) ao longo dos 11 anos, uma taxa média de detecção de 20,1 casos/1000 nascidos vivos para SG e de 5,7 casos/1000 nascidos vivos para SC, com predomínio para população vulnerável. A vulnerabilidade pode ser categorizada em três dimensões: individual- referente a conhecimentos e informações sobre problemas específicos e atitudes para assumirem condutas ou práticas protetoras; social ou coletivo- diz respeito às relações econômicas, de gênero, étnico/raciais, crenças religiosas e exclusão social; programático ou institucional- relacionado aos serviços de saúde e à forma como estes lidam para reduzir contextos de vulnerabilidade, consequentemente, fatores que influenciam no diagnóstico tardio e na baixa adesão ao tratamento (OZELAME JEEP, et al., 2020).

De acordo com a busca ativa dos casos de SG e SC no estudo de Silva NCP, et al. (2021) realizado em Imperatriz-MA, onde foi detectado que a maioria das mulheres participantes eram mães solteiras, pardas, possuíam uma média de idade de 23,6 anos e baixo nível de escolaridade e baixa renda. Dentre elas apenas $76,1 \%$ realizaram o pré-natal de no mínimo seis consultas e apenas $40 \%$ realizaram o tratamento integral (incluindo o parceiro) para sífilis durante o pré-natal. 92,7\% dos recém-nascidos apresentavam possível quadro de sífilis congênita. Dentre as entrevistadas $21,8 \%$ anteriormente haviam realizado aborto, quatro entrevistadas apresentavam infecção pelo vírus da imunodeficiência humana.

Para combater a sífilis é necessário atuar em diferentes esferas, de acordo com Silva NCP, et al. (2021), considerou-se um pré-natal adequado os que foram realizados no mínimo seis consultas e que obteve um tratamento para sífilis em até um mês ante do parto com administração de Penicilina G benzatina 2.400.000 UI, para sífilis primária e penicilina G benzatina 2.400.000 UI, por três semanas na sífilis latente.

Através do pré-natal assistido e feito de maneira adequada, é possível detectar a sífilis, tratá-la de forma a evitar complicações para a mãe e para o bebê, no entanto é necessário que tanto gestante quanto seu parceiro de vida sejam tratados adequadamente com o protocolo correto de medicação. Na prática, para diminuir a sífilis congênita em nível Brasil ou até mesmo mundial, deve-se buscar as possíveis falhas que podem estar ocorrendo na atenção primária assistencial do pré-natal, e cumprir todos os protocolos do Ministério da Saúde da detecção, diagnóstico até o tratamento da sífilis, de modo a aumentar o acesso das gestantes aos serviços de saúde, oferecer treinamento de capacitação e qualificação aos profissionais de saúde a realizar o teste rápido nas unidades além de atividades educativas para conscientizar as gestantes e seus parceiros do valor do pré-natal para a tríade mãe-filho-pai (HORTA HHL, et al., 2017).

\section{DISCUSSÃO}

O estudo de Farias RO, et al. (2020) relata que se evidenciou, um aumento significativo do número de casos notificados de sífilis gestacional nos últimos 13 anos, consequentemente uma alta porcentagem de óbitos infantis em decorrência da doença, e que estão relacionados não somente a falta de acompanhamento pré-natal, mas também a uma inadequada assistência durante toda a gestação. Com isso evidenciasse que com essa situação, mais esforços devem ser feitos para que se possa ter um diagnóstico precoce e tratamento adequado das gestantes, e consequentemente evitando a transmissão vertical, pois em um futuro não muito distante as crianças poderão nascer com sífilis sem que tenham uma chance de melhor tratamento. É de suma importância que a gestante tenha um acompanhamento na assistência primária no intuito de diagnosticar precocemente e aderir o tratamento a fim de evitar transmissão mãe-filho.

Os estudos de Barbosa DFR, et al. (2020) e Nunes IR, et al. (2020) foram estudos de base ecológica realizados através do SINAN, onde podemos observar e constatar que quanto menor a instrução populacional maior os casos de sífilis e sífilis congênita. Enquanto no estudo de Barbosa DFR, et al. (2020) demonstrava a população da cidade de Maceió que houve apenas 119 casos de sífilis congênita no ano de 2019. O estudo de Nunes IR, et al. (2020) demonstrava que a população do estado do Piauí onde 9 a cada 1000 nascidos vivos apresentavam casos de sífilis congênita.

Já os estudos de Saback MC, et al. (2019) e Aleixo FC, et al. (2019), foram pesquisas quantitativas realizadas em hospitais públicos. Enquanto o estudo de Aleixo FC, et al. (2019), retratou os casos de sífilis congênita em 312 neonatos no Pará e dentre eles ainda houve 22 casos de infecção por exposição ao HIV. 
O estudo de Saback MC, et al. (2019) retratou 277 neonatos com VDRL reagente apresentando um possível caso de sífilis congênita, nestes dois estudos podemos constatar que um pré-natal ineficaz põe a vida do recém-nascido em exposição a riscos de infecções e outras patologias que poderiam anteriormente serem descartadas, se houvessem tratamento e acompanhamento efetivos.

Com a complementação do estudo de Alves PIC, et al. (2020), a sífilis é uma doença infecciosa crônica que está há séculos na humanidade, é considerada maior doença sexualmente transmissível depois da Síndrome da Imunodeficiência Adquirida (AIDS), podendo acometer todo o núcleo familiar. Os estudos de Ozelame JEEP, et al. (2020), Alves PIC, et al. (2020) e Silva NCP, et al. (2021) evidenciaram que a vulnerabilidade tem os mais diversos aspectos que produzem o adoecimento. E que a causa da Sífilis Gestacional é multifatorial e que as ações de controle da sífilis não devem estar centradas apenas na gestante infectada, mas em qualquer cidadão exposto a condições que o torne vulnerável à ocorrência da doença.

No estudo de Araujo SR, et al. (2020) demonstra o sofrimento apresentado pelas mães após descobrirem que os seus recém-nascidos necessitam ficar um período internado na maternidade para realizar o tratamento para sífilis congênita, tempo este que poderia ser evitado com um pré-natal e tratamento adequado. Enquanto os estudos de Macêdo VC, et al. (2020) e de Guanabara MAO, et al. (2017), complementa na questão da desigualdade associada a fatores sociais, econômicos, de infraestrutura e de acesso aos serviços de saúde na atenção básica, assim retardaram o diagnóstico e, consequentemente desencadeia, o tratamento apropriado para a sífilis.

Entretanto no estudo de Guanabara MAO, et al. (2017), corrobora nas questões tecnológicas, onde a gestante com sífilis não tem acesso às diferentes tecnologias para prevenção e controle da Sífilis Gestacional. Ressalta também que a consulta pré-natal ocorre rapidamente, não oferecendo uma boa interação entre o profissional e a mulher, comprometendo o acolhimento e o vínculo, estratégias necessárias à boa qualidade da assistência na consulta. Os profissionais da saúde devem estar treinados para que possam oferecer informações adequadas sobre a doença, maneira de diagnóstico, de tratamento e até mesmo reabilitação em casos mais graves de desenvolvimento da doença.

Sendo que no estudo de Figueiredo DCMM, et al. (2020) se faz necessário a ampliação da oferta dos testes rápidos para a população gestante em geral para uma boa cobertura de assistência de pré-natal, assim como a melhoria na sua qualidade, é de fundamental importância para a aplicação correta das condutas profiláticas. Já nos estudos Domingues CSB, et al. (2021), evidenciaram que é primordial a capacitação dos profissionais de saúde quanto às técnicas do manejo da doença, na abordagem de gestantes com sífilis, respeitando as especificidades, culturas, religião, socioeconômico e dificuldades vivenciadas para o seguimento adequado do tratamento.

No estudo de Pereira BB, et al. (2020) complementa-se com a importância da equipe de enfermagem na realização do pré-natal e do teste rápido da sífilis. Onde desenvolvem papel fundamental na implementação de prevenção e promoção de medidas que atuem de forma adequada no enfrentamento desta patologia para a gestante, terapêutica essa que deve ser seguida para evitar complicações.

No artigo de Gomes NS, et al. (2021), o estudo revelou que as gestantes e seus familiares têm conhecimentos limitados acerca da Sífilis, e a não realização do tratamento por parte dos parceiros e o conhecimento limitado por parte dos profissionais de saúde. Sendo um achado preocupante dada a gravidade da doença, mas explicável, por muitas vezes a doença apresenta sinais e sintomas que podem passar despercebidos, o que dificulta o diagnóstico.

Ainda no estudo de Gomes NS, et al. (2021), um dos motivos que leva a população a desconhecer a sífilis é a forma como a doença se apresenta clinicamente, pois culturalmente o processo de adoecer é envolvido de questões subjetivas diferenciadas por uma linguagem própria determinada pelos sinais e sintomas das doenças, precisam-se desenvolver atividades educativas sobre a doença para que a comunidade abranja seus conhecimento sobre a mesma, fazendo com que torne-se mais fácil reconhecer sintomas, fazendo com que o diagnóstico seja preciso e precoce. 
O estudo Souza MHT e Beck EQ (2019) enfatiza que há falta de aconselhamento às gestantes a respeito da doença durante a realização do pré-natal, indicando falhas no diagnóstico e intervenção do agravo. A complexidade do tratamento para sífilis congênita, somada ao desconhecimento sobre a doença, pode explicar a limitação das gestantes quanto à terapêutica adequada. Lembrando que o tratamento para sífilis congênita é complexo, com um protocolo elaborado pelo Ministério da Saúde (MS), estabelecido de acordo com a sorologia da mãe, dias de vida e resultados de exames do recém-nascido. Ressalta-se que as gestantes acometidas pela sífilis podem ter complicações como abortamento tardio que acontece a partir do quarto mês da gestação, natimortos, hidropisia fetal e ainda parto prematuro.

De acordo com estudos sobre a temática fetos abortados com nove semanas de gestação que apresentavam o treponema ao exame histopatológico, indicavam que abortamentos precoces poderiam ter sido causados por sífilis. Percebe-se que é fundamental a intervenção da equipe de saúde agindo em prol da comunidade identificando o nível de conhecimento sobre a sífilis da população, através de rodas de conversas e de pesquisa ativa na comunidade. Podendo ainda, realizar ações educativas por meios de palestras em grupos, uma vez ao mês, de forma a orientá-los sobre sintomas, formas de transmissão e, induzir atitudes e práticas saudáveis para a prevenção da sífilis e o uso correto do preservativo para evitar o contágio (SOUZA MHT e BECK EQ 2019).

Reforça-se a importância de sensibilizar as gestantes e os parceiros acerca da necessidade do tratamento adequado da sífilis durante as consultas de pré-natais, assim como orientar sobre as consequências do não tratamento dessas infecções para a saúde materna e infantil. É necessário estimular a criação de grupos terapêuticos com as gestantes para maior vínculo e abordagem aos diferentes fatores que influenciam o seguimento clínico dos casos de sífilis congênita com ênfase no processo de educação em saúde. (SOUZA MHT e BECK EQ 2019).

\section{CONSIDERAÇÕES FINAIS}

No Brasil, a sífilis é uma doença que representa um grave problema de saúde pública, devido à falta de informação e a dificuldade a assistência ao pré-natal, causa um elevado índice de sífilis congênita (SC). Com isso, se faz necessário o comprometimento de todos os profissionais de saúde quando o objeto é a saúde da população gestante e incentivar mais pesquisas para o monitoramento das Doenças Sexualmente Transmissíveis (DST), objetivando a redução ou até mesmo a erradicação da sífilis congênita. Há uma necessidade de implantar melhorias no serviço de saúde, aumentando a divulgação da doença, favorecendo a prevenção primária da sífilis congênita. Existe necessidade de prestar esclarecimentos e retirar todas as dúvidas das gestantes sobre a gravidade dos casos de sífilis congênita, bem como as formas de transmissão e contagio. A fim de reduzir o número de casos de SC no Brasil.

\section{REFERÊNCIAS}

1. ALEIXO FC, et al. Ocorrência de sífilis congênita e exposição ao HIV em neonatos atendidos em um hospital materno-infantil. Revista Eletrônica Acervo Saúde, 2019; 29(1): e1028.

2. ALVES PIC, et al. Evolução temporal e caracterização dos casos de sífilis congênita em Minas Gerais, Brasil, 20072015. Ciênc. Saúde Colet., 2020; 25(8): 2949-2960.

3. ARAUJO EC et al. Avaliação do pré-natal quanto à detecção de sífilis e HIV em gestantes atendidas em uma área rural do estado do Pará, Brasil. Rev Pan-Amaz Saude. 2018; 9(1): 1-8.

4. ARAUJO SR, et al. A vivência das mães frente a ocorrência de sífilis congênita em seus filhos. Revista Eletrônica Acervo Saúde, 2020; 42(1): e2760.

5. ARRUDA LR, RAMOS ARS. Importância do diagnóstico laboratorial para a sífilis congênita no pré-natal. JMPHC| Journal of Management \& Primary Health Care, 2020; 12: 1-18.

6. BARBOSA DFR, et al. Perfil epidemiológico da sífilis congênita em gestantes no município de Maceió. Revista Eletrônica Acervo Saúde, 2020; 12(11): e4881.

7. BRASIL. Manual do Ministério da Saúde. Secretaria Nacional de Vigilância em Saúde. Programa de DST/AIDS. Boletim Epidemiológico AIDS e DST, Brasília. Ano 2015. Ed 4, v. 1, Disponível em: http://www.aids.gov.br/ptbr/pub/2015/boletim-epidemiologico-hivaids-2015. Acesso em: 22 de abril de 2020. 
8. CAVALCANTE PAM, et al. Sífilis gestacional e congênita em Palmas, Tocantins, 2007-2014. Epidemiologia e Serviços de Saúde, 2017; 26(1): 255-264.

9. DOMINGUES CSB, et al. Protocolo Brasileiro para Infecções Sexualmente Transmissíveis 2020: sífilis congênita e criança exposta à sífilis. Epidemiologia e Serviços de Saúde [online], 2021; 30(1): e2020597.

10. FARIAS RO, et al. The reality of 13 years of prenatal care to pregnant women with syphilis in Sergipe state (20072019). DST J. bras. doenças sex. transm., 2020; 31(4): 123- 130.

11. FIGUEIREDO DCMM, et al. Relação entre oferta de diagnóstico e tratamento da sífilis na atenção básica sobre a incidência de sífilis gestacional e congênita. Cadernos de Saúde Pública, 2020; 36(1): e00074519.

12. GOMES NS, et al. "Só sei que é uma doença": conhecimento de gestantes sobre sífilis. Rev. bras. promoç. Saúde, 2021; 34(1): 1-10.

13. GUANABARA MAO, et al. Acesso de gestantes às tecnologias para prevenção e controle da sífilis congênita em Fortaleza-Ceará, Brasil. Rev. salud pública, 2017; 19(1): 73-78.

14. HORTA HHL, et al. Pré-natal do parceiro na prevenção da sífilis congênita. Rev. APS. 2017; $20(4): 623$ - 627.

15. LAFETÁ KRG, et al. Sífilis materna e congênita, subnotificação é difícil controle. Revista Brasileira de Epidemiologia, 2016; 19(1): 63-74.

16. MACÊDO VC, et al. Sífilis na gestação: barreiras na assistência pré-natal para o controle da transmissão vertical. Cad. Saúde Colet., 2020; 28(4): 518-528.

17. MAGALHÃES DMS, et al. Sífilis materna e congênita: ainda um desafio. Cadernos de Saúde Pública, 2013; 29(6): 1109-1120.

18. NUNES IR, et al. Sífilis Congênita: Caracterização epidemiológica no estado do Piauí, Brasil. Revista Eletrônica Acervo Saúde, 2020; 50(1): e755.

19. OZELAME JEEP, et al. Vulnerabilidade à sífilis gestacional e congênita: uma análise de 11 anos. Rev. enferm. UERJ, 2020; 28(1): e50487.

20. PEREIRA BB, et al. Realização de testes rápidos de sífilis em gestantes por enfermeiros da atenção básica. Rev. enferm. UFSM, 2020; 10(1): e82.

21. SABACK MC, et al. Perfil epidemiológico da sífilis gestacional e congênita na Maternidade Ana Braga - Manaus, Amazonas. Revista Eletrônica Acervo Saúde, 2019; 11(5): e299.

22. SILVA NCP, et al. Sífilis gestacional em uma maternidade pública no interior do Nordeste brasileiro. Femina. 2021; 49(1): 58-61.

23. SOUZA MHT, BECK EQ. Compreendendo a sífilis congênita a partir do olhar materno. Rev. enferm. UFSM. 2019; 9(1): 1-13.

24. SOUZA MT, et al. Revisão integrativa: o que é e como fazer. Einstein (São Paulo). 2010; 8(1): 102-106.

25. SUTO CSS, et al. Assistência pré-natal à gestante com diagnóstico de sífilis. Rev. enferm. atenção saúde, 2016; 5(2): 18-33. 